\title{
Resistive upper critical fields and anisotropy of an electron-doped infinite-layer cuprate
}

\author{
V. P. Jovanović, Z. Z. Li, and H. Raffy \\ Laboratoire de Physique des Solides, Université Paris-Sud, CNRS UMR 8502, 91405 Orsay, France \\ J. Briatico \\ CNRS/THALES, UMR 137, Route D128, 91176 Palaiseau, France
}

\author{
A. A. Sinchenko \\ Moscow Engineering-Physics Institute, 115409 Moscow, Russia and Institute Néel, CNRS and Université Joseph Fourier, \\ Boîte Postale 166, 38042 Grenoble, France \\ P. Monceau \\ Institute Néel, CNRS and Université Joseph Fourier, Boîte Postale 166, 38042 Grenoble, France \\ and Laboratoire National des Champs Magnétiques Intenses, CNRS, Boîte Postale 166, 38042 Grenoble, France
}

(Received 6 March 2009; revised manuscript received 2 June 2009; published 1 July 2009)

\begin{abstract}
We report a transport study down to $2 \mathrm{~K}$ and in high magnetic fields up to $20 \mathrm{~T}$ of a highly $c$-axis-oriented epitaxial thin film of electron-doped "infinite-layer" cuprate superconductor $\mathrm{Sr}_{1-x} \mathrm{La}_{x} \mathrm{CuO}_{2}$ with $T_{c}$ of $26 \mathrm{~K}$. A $16 \mathrm{~T}$ magnetic field perpendicular to the $a b$ plane of the film completely suppresses superconductivity, even at the lowest temperature. Perpendicular magnetic fields shift the resistive transition to lower temperatures, without significant broadening, in a way similar to that seen in conventional superconductors. The upper critical field $H_{c 2 \perp}(T)$ exhibits an almost linear temperature dependence. It is shown that electron-doped infinite-layer cuprate presents three-dimensional superconductivity due to a weaker anisotropy $(\gamma=15)$ and smaller $H_{c 2 \perp}(T)$ values than the ones of hole-doped cuprates.
\end{abstract}

DOI: $10.1103 /$ PhysRevB.80.024501

PACS number(s): 74.78.Bz, 74.25.Fy, 74.25.Op

\section{INTRODUCTION}

Among all the cuprates, the electron-doped (e-doped) $\mathrm{Sr}_{1-x} \mathrm{La}_{x} \mathrm{CuO}_{2}$ (SLCO) compound has the simplest structure, so-called "infinite-layer" structure, where the $\mathrm{CuO}_{2}$ planes are only separated by ( $\mathrm{Sr}, \mathrm{La})$ layers, without intervening charge reservoir blocks. Electron doping is achieved by partial substitution of $\mathrm{Sr}^{2+}$ by $\mathrm{La}^{3+}$ (Ref. 1). A study of this compound can bring new insights on the fundamental properties of cuprates and can allow one to establish which of them are common for hole-doped ( $h$-doped) (Ref. 2$)$ and $e$-doped ones. It is also important to compare the properties of this compound to those of the only well-studied family of $e$-doped cuprates, namely, $\mathrm{Ln}_{2-x} \mathrm{Ce}_{x} \mathrm{CuO}_{4}(\mathrm{Ln}=\mathrm{Nd}, \mathrm{Pr}, \ldots)$. The latter family is structurally more complicated: $\mathrm{Cu}$ sites in adjacent planes do not lie on top of each other along the $c$ axis but on top of centers of $\mathrm{Cu}$ squares. The distance between $\mathrm{CuO}_{2}$ planes is larger and in addition there are also magnetic atoms.

SLCO is very hard to synthesize. To our knowledge there are no single crystals and measurements on highly oriented samples are desirable. Ceramic samples are obtained by high-pressure (2-6 GPa) synthesis. ${ }^{3}$ It is possible to make thin films using epitaxial growth on an adequate substrate ${ }^{4,5}$ but it is still difficult to obtain superconducting stable samples. The highest transition temperature $T_{c}$ of optimally doped SLCO with $x=0.1$ is $43 \mathrm{~K}^{3}$ Various measurements were performed on such ceramic and uniaxially oriented powder samples. Magnetization and penetration-depth measurements suggest three-dimensional (3D) superconductivity (SC). ${ }^{6-8}$ Recent muon spin-rotation ( $\left.\mu \mathrm{SR}\right)$ measurements imply nodeless superconducting gap with a substantial $s$-wave component in the superconducting order parameter, ${ }^{9}$ supporting previously reported results of tunneling, ${ }^{10}$ specific heat, ${ }^{11}$ and small-angle neutron-scattering experiments. ${ }^{12}$ Upon replacing $\mathrm{Cu}$ by $3 \%$ nonmagnetic $\mathrm{Zn}$ impurities no suppression of $T_{c}$ was found while $2 \% \mathrm{Ni}$ impurities nearly suppressed superconductivity of SLCO, ${ }^{13}$ a behavior similar to conventional superconductors, rather than $h$-doped cuprates. Recent scanning tunneling spectroscopy studies of SLCO vortex cores imply that the ground state is not purely superconducting and some competing order may appear. ${ }^{14}$ The best fit to these data is obtained by assuming the coexistence of $d$-wave SC and commensurate spin-density wave. Earlier NMR measurements of SLCO (Refs. 15 and 16) show spin dynamics similar to that of $h$-doped cuprates, implying pairing possibly mediated by antiferromagnetic spin fluctuations, as some authors thought to coexist with SC in the other class of $e$-doped $\mathrm{Ln}_{2-x} \mathrm{Ce}_{x} \mathrm{CuO}_{4}$ cuprates. ${ }^{17}$ The NMR data ${ }^{15}$ can be interpreted in terms of $d$-wave pairing and not in terms of isotropic $s$ wave. The question of pairing symmetry is still not solved. Although it has a very simple structure, there are no band-structure calculations for $e$-doped SLCO, as far as we know.

There are only very few transport measurements on SLCO samples. Of all the transport properties, mainly inplane resistivity vs temperature was measured as a part of ceramic or thin-film sample characterization., ${ }^{1,5,18}$ It was shown that optimally doped films on $\mathrm{DyScO}_{3}$ substrate $\left(T_{c}\right.$ $\approx 41 \mathrm{~K})$ do not display a Fermi-liquid quadratic behavior $\rho \propto T^{2}$ (Ref. 18) observed for the other $e$-doped family. ${ }^{19,20} \mathrm{In}$ a previous paper, ${ }^{21}$ we reported, for epitaxial thin films deposited on $\mathrm{KTaO}_{3}$ substrates, a nonmonotonic temperature dependence of the Hall effect with sign changes, indicating 
the presence of two types of charge carriers with different mobilities and we observed a normal-state negative magnetoresistance for the most underdoped films in magnetic fields parallel to the $a b$ plane of the film, $\mathbf{H}_{\|}$. In this paper we report the results of transport measurements performed on a highly $c$-axis-oriented $x=0.12$ SLCO thin film under high magnetic fields up to $20 \mathrm{~T}$. After examining the behavior of the temperature dependence of the resistivity of the film, we will present and discuss the effect on the resistive transition of a magnetic field applied perpendicular to the film $a b$ plane, $\mathrm{H}_{\perp}$, the temperature dependence of the upper critical field, $H_{c 2 \perp}(T)$, the angular dependence of the mixed-state magnetoresistance (AMR), the anisotropy, and the dimensionality of SC. We will compare these results to those obtained on ceramic SLCO, $h$ - and $e$-doped cuprates.

\section{EXPERIMENTAL}

Single phase $c$-axis-oriented epitaxial thin films of $\mathrm{Sr}_{0.88} \mathrm{La}_{0.12} \mathrm{CuO}_{2}$ were deposited on a heated $\mathrm{KTaO}_{3}$ substrate by $\mathrm{rf}$ magnetron sputtering technique, used also for depositing $\mathrm{Bi}_{2} \mathrm{Sr}_{2-x} \mathrm{La}_{x} \mathrm{CuO}_{6}$ thin films. ${ }^{22} \mathrm{~A}$ detailed preparation description is given in Ref. 5. It is emphasized, as in Ref. 4, that the choice of the substrate is essential for obtaining superconducting samples, as well as is an oxygen reduction step since the as-prepared films are insulating. The films were characterized by $x$-ray diffraction spectra $^{5}$ which show that our films are epitaxial, single phase, and highly $c$-axis-oriented with a mosaicity of $0.1^{\circ}$, a value even better than that of $\mathrm{Bi}_{2} \mathrm{Sr}_{2-x} \mathrm{La}_{x} \mathrm{CuO}_{6}$ thin films. ${ }^{22}$ The composition of SLCO deposits was checked by inductive-coupled plasma spectroscopy, giving $x=0.12$. The highest $T_{c}$ obtained in this way for now is $26 \mathrm{~K}$, the sample still being underdoped.

The transport measurements were performed using a classical four-probe dc method. SLCO thin films were patterned, using optical lithography and chemical etching, in a standard six-contact resistivity bridge, the track being $0.35 \mathrm{~mm}$ wide and $1.04 \mathrm{~mm}$ long. Measurements of $\rho(T, H)$ under perpendicular or parallel magnetic fields with $H \leq 5 \mathrm{~T}$ were first made in a Quantum Design MPMS apparatus on several samples which gave similar results. The measurements of $\rho(H, T)$ under high magnetic fields up to $20 \mathrm{~T}$, reported here, were performed in the Laboratoire National des Champs Magnétiques Intenses at Grenoble and they were made on a thin film, $50 \mathrm{~nm}$ thick, with tetragonal lattice parameters $a$ $=3.988 \AA$ and $c=3.397 \AA$ and with $T_{c}$ equal to $26 \mathrm{~K}$. For both experiments the lower-temperature limit was $2 \mathrm{~K}$. In the latter case, the sample was mounted on a rotating plate with the rotation axis along the film $a$ axis and parallel to the direction of the current. In the measurements, the magnetic field was always perpendicular to the current (typically $10 \mu \mathrm{A}$ or $60 \mathrm{~A} / \mathrm{cm}^{2}$, a value sufficiently small to avoid self-heating or nonohmic behavior). The measurements of the mixed-state magnetoresistance $\rho(H, T)$ were conducted in $H_{\perp}(H \| c)$ and $H_{\|}(H \| b)$ at given $T$, as well as the measurements of the angular dependence of the magnetoresistance, AMR, $\Delta \rho(\theta, T, H)$, for given $T$ and $H$, where $\pi / 2-\theta$ is the angle between the film $c$ axis and $H$.

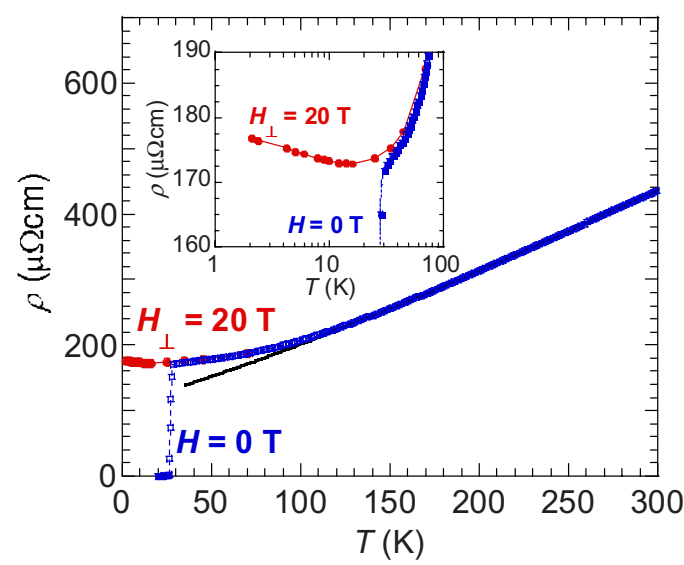

FIG. 1. (Color online) $\rho(T)$ in $H_{\perp}=0$ and $20 \mathrm{~T}$. In the inset the transition region is shown in a semilog plot. The solid line represents the fits valid for $145<\mathrm{T} \leq 300 \mathrm{~K}$ (see text).

\section{RESULTS AND DISCUSSION}

\section{A. Temperature dependence of the resistivity}

Let us consider first the temperature dependence of the resistivity, $\rho(T)$, in the absence of a magnetic field and in a magnetic field $H_{\perp}=20 \mathrm{~T}$ (Fig. 1). In zero magnetic field, the $\rho(T)$ curve exhibits a monotonous decrease with decreasing temperature. The empirical temperature dependence often taken for $h$-doped cuprates, $\rho(T)=\rho_{0}+C T^{n},{ }^{23}$ where $\rho_{0}$ is a residual resistivity and $C$ is a constant, can be best adjusted to the data for $T>140 \mathrm{~K}$ (Ref. 24$)$ and $n \approx 1.18$ (Table I), a value lower than previously reported $(n \approx 1.5)$ for the optimally doped SLCO films. ${ }^{18}$ Note that in the case of underdoped $\mathrm{Bi}_{2} \mathrm{Sr}_{2-x} \mathrm{La}_{x} \mathrm{CuO}_{6}$ a value $n<1$ and a negative curvature of $\rho(T)$ in this temperature region was generally found. ${ }^{23}$

Equivalently, in the same temperature range, the data can be fitted by a polynomial law of the form $\rho_{0}+A T+B T^{2}$, where $A$ and $B$ are constant. For $T>145 \mathrm{~K}$, the best fit is obtained with the parameters: $A \approx 1 \mu \Omega \mathrm{cm} / \mathrm{K}$ and $B$ $\approx 0.5 \mathrm{n} \Omega \mathrm{cm} / \mathrm{K}^{2}$ (Table I). The linear term is dominant in contrast to $\mathrm{Nd}_{2-x} \mathrm{Ce}_{x} \mathrm{CuO}_{4}$ (NCCO) where a quadratic behavior was reported in a large temperature interval. ${ }^{19,20}$ Supposing the linear term would come from electron-phonon $(e-p h)$ interactions, then the coefficient $A$ of this linear term can be estimated to be $0.9 \mu \Omega \mathrm{cm} / \mathrm{K}$ using Ziman's formula $\rho_{\text {phonon }}(T)=8 \pi^{2} k_{B} \lambda T /\left(\hbar \omega_{p}^{2}\right)$, valid at high temperatures,

TABLE I. Parameter values of different fits of $\rho(T)$ given in Fig. 1 .

\begin{tabular}{cccc}
\hline \hline & \multicolumn{3}{c}{$\rho(T)=\rho_{0}+C T^{n}$} \\
& $\rho_{0}$ & $C$ \\
$(\mu \Omega \mathrm{cm})$ & $\left(\mathrm{n} \Omega \mathrm{cm} / \mathrm{K}^{n}\right)$ & $n$ \\
\hline$T>140 \mathrm{~K}$ & 113 & 381 & 1.18 \\
& & $\rho(T)=\rho_{0}+A T+B T^{2}$ & \\
& $\rho_{0}$ & $A$ & $B$ \\
& $(\mu \Omega \mathrm{cm})$ & $(\mu \Omega \mathrm{cm} / \mathrm{K})$ & $\left(\mathrm{n} \Omega \mathrm{cm} / \mathrm{K}^{2}\right)$ \\
\hline$T>145 \mathrm{~K}$ & 95 & 1 & 0.5 \\
\hline \hline
\end{tabular}


given in Ref. 25. The plasma frequency $\hbar \omega_{p} \approx 2 \mathrm{eV}$ was calculated from the magnetic penetration depth $\lambda_{a b}$ $\approx 100 \mathrm{~nm},{ }^{26}$ measured by $\mu$ SR on powder SLCO samples with $x=0.125$. It was assumed that SLCO has a similar $e$-ph coupling constant $\lambda=0.9$ to $\mathrm{NCCO}{ }^{25}$ Although the calculated value of $A$ is close to the corresponding one obtained by the described polynomial fit, it cannot be excluded that scattering mechanisms other than $e-p h$ scattering come into play to explain this linear term. It is very difficult to conclude only from resistivity measurements which is the role of $e$-ph scattering and also how much holes, detected in our Hall-effect measurements, ${ }^{21}$ contribute to the resistivity. To help to resolve some of these questions, other studies such as tunneling or Raman spectroscopy are needed.

Finally, one remark about $\rho(T)$ in zero field should be made. For $h$-doped superconductor it is possible to determine the pseudogap temperature $T^{*}$ from $\rho(T)$ curve, as the temperature below which the resistivity starts to decrease faster than linearly. ${ }^{23}$ The $\rho(T)$ variation in SLCO does not have such a feature and $T^{*}$ cannot be determined by a similar criterion. To prove directly the existence of the pseudogap in the normal state of SLCO, tunnelling or photoemission spectroscopy has to be performed.

Figure 1 also shows that SC in the sample is completely suppressed in a perpendicular magnetic field of $20 \mathrm{~T}$ and it is seen that below $T \approx 15 \mathrm{~K}$ an upturn in $\rho(T)$ occurs (inset of Fig. 1). The resistivity diverges logarithmically below $10 \mathrm{~K}$. Similar trend was observed in the cases of $h$-doped $\mathrm{La}_{2-x} \mathrm{Sr}_{x} \mathrm{CuO}_{4}$ (LSCO) (Ref. 27) and $\mathrm{Bi}_{2} \mathrm{Sr}_{2-x} \mathrm{La}_{x} \mathrm{CuO}_{6}$ (Ref. 28) cuprates with comparable $T_{c}$. It was suggested there that $\ln (1 / T)$ temperature dependence of in-plane resistivity is a consequence of unusual charge localization common in all the cuprates.

\section{B. Resistive transitions in perpendicular magnetic field}

Let us focus now on the most important results of this paper. As shown in Fig. 2, the effect of increasing perpendicular magnetic fields $H_{\perp}$ on the resistive transition is to shift the fairly sharp resistive superconducting transition toward lower temperatures without significant broadening. The weak broadening and the shift of the transition to lower $T$ is also seen in the other class of $e$-doped cuprates, namely, NCCO (Ref. 20) while the behavior of $h$-doped cuprates is very different: the transition is significantly broadened. ${ }^{29,30}$ The $\rho(H, T)$ data in Fig. 2(b) for $H \leq 5 \mathrm{~T}$ were taken in previous measurements. ${ }^{21}$ The resistivity curves $\rho(H, T)$ obtained under high magnetic fields are shown in Fig. 2(a). In Fig. 2(b), the upper critical field, $H_{c 2 \perp}$, is determined at the onset of the transition and in Fig. 2(a) at the midpoint (where half of the normal-state resistivity is reached) because it agrees better with $H_{c 2 \perp}$ determined from Fig. 2(b). The "onset" $H_{c 2 \perp}(T)$ is systematically higher for approximately a constant value than "midpoint" $H_{c 2 \perp}(T)$, since the broadening of the transition is weak at higher fields, as can be easily seen from Fig. 2. The irreversibility field $H_{\text {irr } \perp}$ is determined at the point $\left(T_{\mathrm{irr} \perp}, H_{\mathrm{irr} \perp}\right)$, where the resistive state appears by increasing $H$ or $T$ (typically $0.1 \Omega$ or $0.2 \mu \Omega \mathrm{cm}$, Fig. 2). The temperature dependences of $H_{c 2 \perp}$ and $H_{\text {irr } \perp \text { are plotted }}$
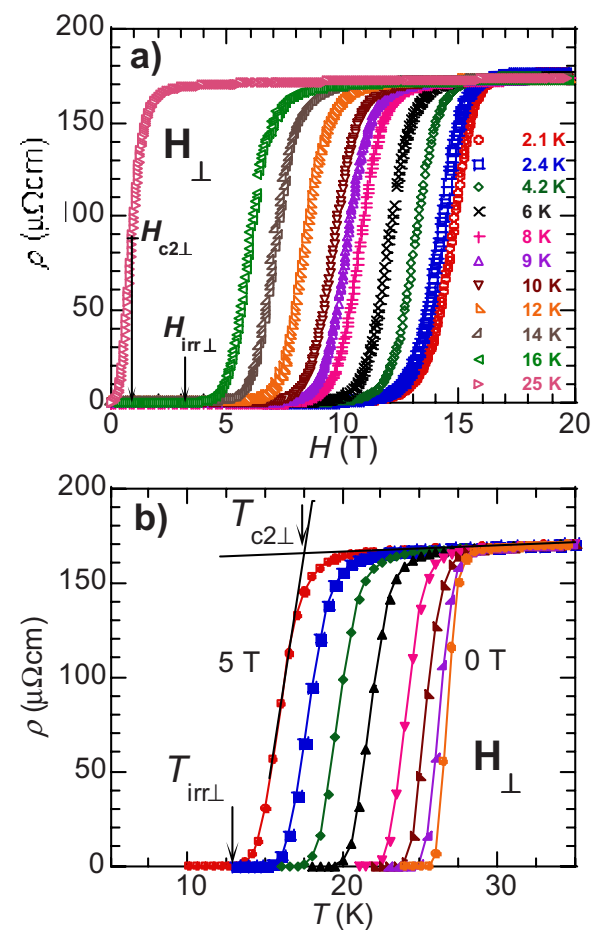

FIG. 2. (Color online) (a) The $\rho\left(H_{\perp}\right)$ curves for different temperatures. (b) $\rho(T)$ for $H=0,0.2,0.5,1,2,3,4,5 \mathrm{~T}$. The arrows indicate how $T_{c 2 \perp}$ and $T_{\text {irr } \perp}$, i.e., $H_{c 2 \perp}$ and $H_{\text {irr } \perp}$, are determined.

in Fig. 3. The quantities marked with the filled circles and upward triangles are determined from Fig. 2(a) while the ones with squares and downward triangles from Fig. 2(b). The matching between these quantities is satisfactory.

\section{Perpendicular upper critical fields}

Now we will discuss the behavior of $H_{c 2 \perp}(T)$ in some detail. The $H_{c 2 \perp}(T)$ of our SLCO is almost linear in $T$ in the whole temperature range and its behavior is markedly differ-

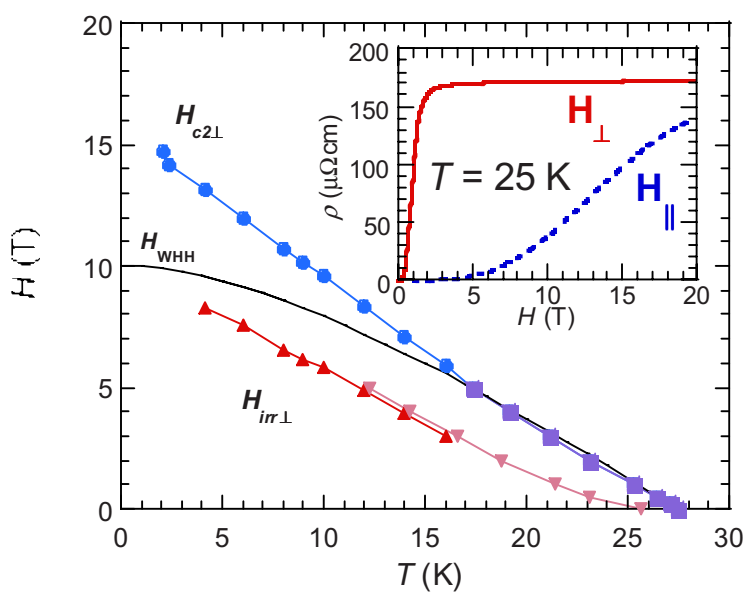

FIG. 3. (Color online) $H_{c 2 \perp}(T)$ and $H_{\text {irr } \perp}(T)$ as determined from Fig. 2. Filled circles and upward triangles correspond to high-field measurements in Fig. 2(a) while squares and downward triangles to low-field ones in Fig. 2(b). Solid $H_{\mathrm{WHH}}$ line is the calculated WHH behavior from the slope of $H_{c 2 \perp}(T)$ close to $T_{c}$. The inset shows $\rho\left(H_{\perp}\right)$ and $\rho\left(H_{\|}\right)$at $25 \mathrm{~K}$ indicating the anisotropy $\gamma$ to be around 15 . 
ent from the one given by the Werthamer-HelfandHohenberg (WHH) theory ${ }^{31}$ (solid $H_{\mathrm{WHH}}$ line in Fig. 3), which works very well for conventional superconductors with weak electron-phonon coupling. In the WHH theory $H_{c 2 \perp}(T)$ is linear in $T$ at high temperatures (close to $T_{c}$ ). At low $T$ there is a characteristic saturation of $H_{c 2 \perp}(T)$. In contrast, a positive curvature and a divergence at low $T$ of $H_{c 2 \perp}(T)$ is a common feature for different $h$-doped cuprates. ${ }^{30,32-34}$ Our SLCO film does not have a saturation nor a divergence of $H_{c 2 \perp}(T)$ at low $T$. This result does not depend on whether $H_{c 2 \perp}$ is determined at the midpoint or onset of the superconducting transition, unlike for most $h$-doped cuprates. As a matter of fact, it was emphasized in Ref. 30 that the positive curvature of the resistive $H_{c 2 \perp}(T)$ of cuprates depends on how the normal-state resistivity and $H_{c 2 \perp}$ values are determined. In effect, considering optimally doped (OPT) cuprates with different anisotropy, it appears that, if $H_{c 2} \perp$ is taken at the midpoint, OPT $\mathrm{Bi}_{2} \mathrm{Sr}_{2} \mathrm{CaCu}_{2} \mathrm{O}_{y}$ (Bi-2212, highly anisotropic) shows an upward curvature stronger than the one of OPT LSCO (moderately anisotropic) while $H_{c 2 \perp}(T)$ for OPT $\mathrm{YBa}_{2} \mathrm{Cu}_{3} \mathrm{O}_{y}$ (YBCO, weakly anisotropic) is almost linear in $T$. If $H_{c 2 \perp}$ is taken at $90 \%$ of the normal-state resistivity then only $\mathrm{Bi}-2212$ shows an upward curvature. Thus it is argued that the curvature of $H_{c 2 \perp}(T)$ is higher for higher anisotropy. The same argument applies if the anisotropy of the cuprate is increased by decreasing the doping. ${ }^{30}$ In the case of $e$-doped NCCO an underdoped film has a stronger upward curvature than an OPT film while $H_{c 2 \perp}(T)$ is linear in $T$ for overdoped films. ${ }^{20}$ Similar observation was made for LSCO in Ref. 30, where it was concluded that such doping dependence of the curvature of $H_{c 2 \perp}(T)$ simply arises from a decrease in the anisotropy which accompanies increasing carrier concentration. If this reasoning is correct and can be applied to SLCO, then the high carrier concentration of SLCO indicated by previous measurements of the Hall constant which has a very small magnitude, ${ }^{21}$ implies a moderate anisotropy and may explain the absence of positive curvature of $H_{c 2 \perp}(T)$. Indeed, we estimated a smaller or moderate value of the anisotropy of our SLCO film (see the text further below). In fact, both higher carrier concentration and moderate anisotropy of SLCO are to be related to the concentration of conducting planes higher than in other cuprates.

The behavior of resistive $H_{c 2 \perp}(T)$ in $h$-doped cuprates fueled a large debate about its interpretation. One may wonder if $H_{c 2 \perp}(T)$ determined here is really a transition field or if it is only the field which breaks the long-range phase coherence. The absence of broadening of the resistive transition under magnetic field is reminiscent of the behavior of conventional superconductors. However it has been pointed out in Ref. 35 that it may not be simply associated to a superconducting normal-state transition at $H_{c 2 \perp}$ and some shortrange SC order may subsist in the sample above the transition. So overdoped $\mathrm{Tl}_{2} \mathrm{Ba}_{2} \mathrm{CuO}_{6+d}$ single crystals have abrupt resistive transitions, yielding $H_{c 2 \perp}(T)$ curves independent of the criterion of determination but they present a strong positive curvature and a low $T$ divergence not seen for SLCO. In contrast specific-heat measurements on similar Tl-2201 samples gave higher, linear $H_{c 2 \perp}(T)$ and the authors ${ }^{35}$ concluded that the specific-heat anomalies measured under mag- netic fields showed the signs of the presence of strong thermal fluctuations. ${ }^{36}$ No thermodynamic measurements of the SC transition have been performed on SLCO yet. The high carrier concentration and the 3D behavior in this system (see Sec. III E below) may suggest that fluctuations are less important than in most $h$-doped cuprates.

\section{Irreversibility fields}

The irreversibility field $H_{\text {irr } \perp}(T)$ (Fig. 3) also presents some unusual features for a cuprate superconductor. A strong upward curvature of $H_{\mathrm{irr} \perp}(T)$ is what one observes for an $h$-doped cuprate. ${ }^{30,33,34}$ The high-temperature dependence of $H_{\text {irr } \perp}(T)$ of our SLCO film shows a positive curvature and it smoothly transforms into a linear $T$ dependence as the temperature is lowered. It does not exhibit the so-called decoupling transition from the empiric power-law behavior at low fields, $H_{\text {irr } \perp}(T)=H_{0}\left(1-T / T_{c}\right)^{n}$, to an exponential behavior $\propto \exp (1 / T)$ at high fields. Such decoupling transition occurs in quasi-two-dimensional (2D) superconductors such as Bi-2212. ${ }^{37}$ In weakly anisotropic systems such as YBCO it does not exist due to the stronger coupling between $\mathrm{CuO}_{2}$ planes. The fit of the above power law to low-field data (marked with downward triangles in Fig. 3) gives $n=1.4$ and $H_{0}=12.4 \mathrm{~T}$ for $T_{c}=25.7 \mathrm{~K}$. The value of $n$ is similar to the one of YBCO $(n=1.5)$ (Ref. 38), pointing again to weaker anisotropy of SLCO. This was previously emphasized by Kim et al. ${ }^{7}$ They found that $n=1.67$ for OPT SLCO-oriented powder samples in the same field range. The region of vortex liquid is narrow (Fig. 3), unlike for $h$-doped cuprates, where pronounced upward curvature is stronger for the systems with higher anisotropy. ${ }^{30}$ Although the behavior of $H_{c 2 \perp}(T)$ of SLCO may have some similitude with the one of $h$-doped cuprate with moderate $\gamma$, the behavior of $H_{\mathrm{irr} \perp}(T)$ is in general very different.

\section{E. Anisotropy and 3D behavior}

In order to determine the anisotropy of this SLCO film, measurements of the magnetoresistance in perpendicular field, $\rho\left(H_{\perp}\right)$, and in carefully aligned parallel magnetic field, $\rho\left(H_{\|}\right)$, were carried at $25 \mathrm{~K}$ (see the inset of Fig. 3). An evaluation of the anisotropy ratio $\gamma=\xi_{a b} / \xi_{c}$ was made from the ratio $H_{\|}^{a} / H_{\perp}^{a}$ for which $\rho\left(H_{\|}^{a}=20 \mathrm{~T}\right)=\rho\left(H_{\perp}^{a}\right)$. It is found to be close to 15 . The linear extrapolation of $H_{c 2 \perp}(T)$ to $T$ $=0 \mathrm{~K}$ yields $H_{c 2 \perp}(0) \approx 16 \mathrm{~T}$ and a zero-temperature inplane coherence length, $\xi_{a b}(0)$, around $45 \AA$. From the extrapolated value of the perpendicular upper critical field, $H_{c 2 \perp}(0)$, and supposing that the anisotropy parameter $\gamma$ keeps the same value at $T=0$, we estimate the in-plane upper critical field to be $H_{c 2 \|}(0)=\gamma H_{c 2 \perp}(0)=240 \mathrm{~T}$. This value appears to be significantly larger than the Pauli paramagnetic limit of $160 \mathrm{~T}$, which was estimated from the well-known Clogston formula using the value of the superconducting gap $\Delta=13 \mathrm{meV} .{ }^{10}$ If so, superconductivity at low temperatures would be limited by normal-state paramagnetism, which was also one of the conclusions of magnetization measurements. ${ }^{8}$ It is to be noted that a low-temperature paramagnetic limitation of the in-plane magnetic field has been reported for $\mathrm{Bi}$ 2201 (Ref. 39) and for $\mathrm{NCCO},{ }^{40}$ systems where the upper 
TABLE II. Superconducting transition temperature $T_{c}$, lattice parameters $a$ and $c$, upper critical field $H_{c 2 \perp}(0)$, in-plane $\xi_{a b}(0)$ and out-of-plane $\xi_{c}(0)$ coherence lengths, and anisotropy ratio $\gamma$ of the SLCO sample.

\begin{tabular}{ccccccc}
\hline \hline $\begin{array}{c}T_{c} \\
(\mathrm{~K})\end{array}$ & $\begin{array}{c}a \\
(\AA)\end{array}$ & $\begin{array}{c}c \\
(\AA)\end{array}$ & $\begin{array}{c}H_{c 2 \perp}(0) \\
(\mathrm{T})\end{array}$ & $\begin{array}{c}\xi_{a b}(0) \\
(\AA)\end{array}$ & $\begin{array}{c}\xi_{c}(0) \\
(\AA)\end{array}$ & $\gamma$ \\
\hline 26 & 3.988 & 3.397 & 16 & 45 & 3 & 15 \\
\hline \hline
\end{tabular}

critical-field values are not too high to be measured. From $\gamma$ and $\xi_{a b}(0)$ the interplane coherence length $\xi_{c}(0)$ is calculated to be of the order of $3 \AA$ for our SLCO film. The different film parameters are summarized in Table II. Taking the condition for 2D to 3D crossover $\xi_{c}\left(T^{*}\right)=s / \sqrt{2},{ }^{41}$ where $s$ is the interplane distance (in this case equal to $c$-axis parameter $c$ $\approx 3.4 \AA$ ) and assuming that our estimation of $H_{c 2 \perp}(0)$ and $\gamma$ is correct, we find $\xi_{c}(0)>s / \sqrt{2}$. This implies strong coupling between $\mathrm{CuO}_{2}$ planes and 3D SC. As mentioned in the introduction, magnetization and penetration-depth measurements on optimally doped oriented powder SLCO samples suggested 3D SC $\left[\xi_{c}(0)=5.2 \AA>s\right]$, with $H_{c 2 \perp}(0) \approx 14 \mathrm{~T}$ (or $12 \mathrm{~T}$ in Ref. 8) and an anisotropy ratio $\gamma \approx 9 .{ }^{6-8}$ Compared to the results on our underdoped SLCO film $\left(T_{c}=26 \mathrm{~K}\right.$ and $x$ $=0.12), H_{c 2 \perp}(0)$ extracted from $\lambda(H)$ measurements on the optimally doped polycrystalline samples has the same order of magnitude whereas $H_{c 2 \perp}(T)$ shows a low- $T$ saturation. ${ }^{8}$ The anisotropy of oriented powder samples ${ }^{6}$ is weaker than that of our film, one of the possible reasons being the lower quality of orientation in powder. This can be seen from x-ray diffraction as the rocking curve of the powder ${ }^{6}$ is around ten times wider than that of our film. ${ }^{5}$ For NCCO with $T_{c}$ $=24.4 \mathrm{~K}, H_{c 2 \perp}(0) \approx 7 \mathrm{~T}, \xi_{c} \approx 3 \AA$, and $s=c / 2 \approx 6 \AA$, the coupling between the planes is weaker than for SLCO. ${ }^{19,42}$

Another way to evidence the anisotropy of this SLCO sample is to measure the angular dependence of the magnetoresistance under a constant magnetic field. Figure 4 presents the angular dependence of the resistivity, $\rho(\theta, T)$, at constant temperature ( $T=25 \mathrm{~K}$ at the foot of the superconducting transition), in a constant magnetic field $H=5 \mathrm{~T}$. For $\theta=0^{\circ}$ (parallel field), very low resistivity appears because a field of $5 \mathrm{~T}$ is far from being sufficient to destroy the SC of the sample in this configuration. As the angle increases, the SC is very quickly suppressed and at an angle of around $30^{\circ}$ the normal state is reached. In the inset of Fig. 4, are plotted two sets of data. The first ones, $\rho(H)$, are the data obtained by measuring the resistance as a function of a perpendicular field $(0 \leq H \leq 5 \mathrm{~T})$. The second ones are the magnetoresistance data, given in the main figure, measured vs angle $\theta$ in a constant field, $H_{0}=5 \mathrm{~T}$, and plotted versus an equivalent field $H_{\mathrm{T}}$, given by the anisotropic mass law for a $3 \mathrm{D}$ anisotropic system: ${ }^{43} H_{\mathrm{T}}=5 \mathrm{~T} \cdot\left[\sin ^{2}(\theta)+\cos ^{2}(\theta) / \gamma^{2}\right]^{1 / 2}$.

We take the value $\gamma=15$, obtained as described above, by measuring directly the magnetoresistance in parallel and per-

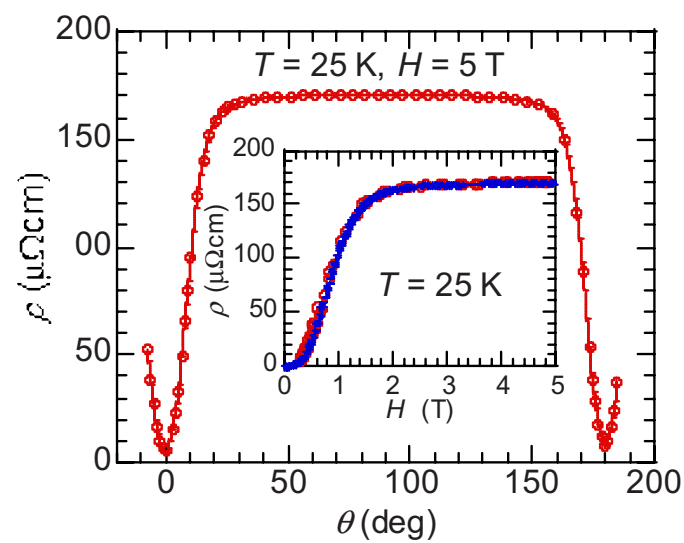

FIG. 4. (Color online) $\rho(\theta)$ for $H=5 \mathrm{~T}$ and $T=25 \mathrm{~K}$. The inset shows the experimental curve $\rho\left(H_{\perp}\right)$ (dots) compared to $\rho\left(H_{\mathrm{T}}\right)$ (open circles), obtained by plotting $\rho(\theta)$ as a function of $H_{\mathrm{T}}$ $=5 \mathrm{~T} \cdot\left[\sin ^{2}(\theta)+\cos ^{2}(\theta) / \gamma^{2}\right]^{1 / 2}$, and taking the value of the anisotropy ratio, $\gamma=15$, determined experimentally (see text).

pendicular fields. The two sets of data lie on a single curve. This demonstrates that the value of $\gamma$ is well determined and that SLCO behaves as a 3D anisotropic superconductor.

\section{CONCLUSION}

Results on transport properties under high magnetic field up to $20 \mathrm{~T}$ of underdoped single phase, $c$-axis-oriented $e$-doped $\mathrm{Sr}_{0.88} \mathrm{La}_{0.12} \mathrm{CuO}_{2}$ epitaxial thin film with $T_{c}=26 \mathrm{~K}$, are reported. The behavior of the normal-state resistivity cannot be simply ascribed to Fermi liquid, as previously reported $^{18}$ for OPT SLCO. The resistive transition to the superconducting state is rather narrow for a cuprate and it hardly broadens upon application of perpendicular magnetic fields, pointing to the weak anisotropy of the system. Interestingly, $H_{c 2 \perp}(T)$ is roughly linear in $T$ in the whole range of temperatures, neither following WHH behavior ${ }^{31}$ of conventional superconductors neither showing a marked upward curvature as $h$-doped cuprates. ${ }^{30}$ Linear extrapolation gives $H_{c 2 \perp}(0) \approx 16 \mathrm{~T}$ and the anisotropy ratio has a moderate value, $\gamma \approx 15$, as confirmed also by angular dependence of resistivity at $T \approx T_{c}$. A $3 \mathrm{D} \mathrm{SC}$ is found down to zerotemperature limit, a result similar to previous reports on magnetic properties of OPT ceramic SLCO ${ }^{6-8}$ There is a strong coupling between the $\mathrm{CuO}_{2}$ planes. As a consequence, the region of vortex liquid is narrow, $H_{\text {irr } \perp}(T)$ being linear in $T$, except in high- $T$ region, where it shows a weak upward curvature. Such behavior is in sharp contrast to that of $h$-doped cuprates. ${ }^{30}$

\section{ACKNOWLEDGMENTS}

We thank F. Bouquet, L. Fruchter, and J. Zasadzinski for helpful discussions. V.J. acknowledges support from the E.C. under an ESRT Marie Curie program No. MEST-CT-2004514307; A.A.S. acknowledges support from INTAS under Grant No. 05-7972. 
${ }^{1}$ M. G. Smith, A. Manthiram, J. Zhou, J. B. Goodenough, and J. T. Markert, Nature (London) 351, 549 (1991).

${ }^{2}$ Hole-doped infinite-layers films have been studied as model systems for understanding the properties of cuprates: see, for instance, C. Aruta et al., Phys. Rev. B 78, 205120 (2008).

${ }^{3}$ G. Er, Y. Miyamoto, F. Kanamuru, and S. Kikkawa, Physica C 181, 206 (1991); N. Ikeda, Z. Hiroi, M. Azuma, M. Takano, Y. Bando, and Y. Takeda, ibid. 210, 367 (1993).

${ }^{4}$ S.-I. Karimoto, K. Ueda, M. Naito, and T. Imai, Appl. Phys. Lett. 79, 2767 (2001); V. Leca, D. H. A. Blank, G. Rijnders, S. Bals, and G. Van Tendeloo, ibid. 89, 092504 (2006).

${ }^{5}$ Z. Z. Li, V. Jovanović, H. Raffy, and S. Megtert, Physica C 469, 73 (2009)

${ }^{6}$ M.-S. Kim, C. U. Jung, J. Y. Kim, J.-H. Choi, and S.-I. Lee, Solid State Commun. 123, 17 (2002).

${ }^{7}$ M.-S. Kim, T. R. Lemberger, C. U. Jung, J.-H. Choi, J. Y. Kim, H.-J. Kim, and S.-I. Lee, Phys. Rev. B 66, 214509 (2002).

${ }^{8}$ V. S. Zapf, N.-C. Yeh, A. D. Beyer, C. R. Hughes, C. Mielke, N. Harrison, M. S. Park, K. H. Kim, and S.-I. Lee, Phys. Rev. B 71, 134526 (2005).

${ }^{9}$ R. Khasanov, A. Shengelaya, A. Maisuradze, D. Di Castro, I. M. Savić, S. Weyeneth, M. S. Park, D. J. Jang, S.-I. Lee, and H. Keller, Phys. Rev. B 77, 184512 (2008).

${ }^{10}$ C.-T. Chen, P. Seneor, N.-C. Yeh, R. P. Vasquez, L. D. Bell, C. U. Jung, J. Y. Kim, M. S. Park, H.-J. Kim, and S.-I. Lee, Phys. Rev. Lett. 88, 227002 (2002).

${ }^{11}$ Z. Y. Liu, H. H. Wen, L. Shan, H. P. Yang, X. F. Lu, H. Gao, M. S. Park, C. U. Jung, and S.-I. Lee, Europhys. Lett. 69, 263 (2005).

${ }^{12}$ J. S. White, E. M. Forgan, M. Laver, P. S. Häfliger, R. Khasanov, R. Cubitt, C. D. Dewhurst, M.-S. Park, D.-J. Jang, H.-G. Lee, and S.-I. Lee, J. Phys.: Condens. Matter 20, 104237 (2008).

${ }^{13}$ C. U. Jung, J. Y. Kim, M.-S. Park, M.-S. Kim, H.-J. Kim, S. Y. Lee, and S.-I. Lee, Phys. Rev. B 65, 172501 (2002).

${ }^{14}$ M. L. Teague, A. D. Beyer, M. S. Grinolds, S. I. Lee, and N.-C. Yeh, Europhys. Lett. 85, 17004 (2009).

${ }^{15}$ G. V. M. Williams, R. Dupree, A. Howes, S. Krämer, H. J. Trodahl, C. U. Jung, M.-S. Park, and S.-I. Lee, Phys. Rev. B 65, 224520 (2002).

${ }^{16}$ T. Imai, C. P. Slichter, J. L. Cobb, and J. T. Markert, J. Phys. Chem. Solids 56, 1921 (1995).

${ }^{17}$ W. Yu, J. S. Higgins, P. Bach, and R. L. Greene, Phys. Rev. B 76, 020503(R) (2007).

${ }^{18}$ S. Karimoto and M. Naito, Physica C 412-414, 1349 (2004).

${ }^{19}$ C. C. Tsuei, A. Gupta, and G. Koren, Physica C 161, 415 (1989).

${ }^{20}$ F. Gollnik and M. Naito, Phys. Rev. B 58, 11734 (1998).

${ }^{21}$ V. Jovanović, Z. Z. Li, F. Bouquet, L. Fruchter, and H. Raffy, J. Phys.: Conf. Ser. 150, 052086 (2009).

${ }^{22}$ Z. Z. Li, H. Rifi, A. Vaurès, S. Megtert, and H. Raffy, Physica C 206, 367 (1993).

${ }^{23}$ Z. Konstantinović, Z. Z. Li, and H. Raffy, Physica C 351, 163 (2001).

${ }^{24}$ At lower temperature, the decrease in $\rho(T)$ is much less rapid, the exponent $n$ taking increasing values with decreasing $T: n$
$=2$ for $75<T<100 \mathrm{~K}$ and $n>2$ below $75 \mathrm{~K}$. Among other possible causes, the influence of disorder, growing with decreasing $T$, precludes a reliable analysis of $\rho(T)$ at low $T$.

${ }^{25}$ N. Tralshawala, J. F. Zasadzinski, L. Coffey, and Q. Huang, Phys. Rev. B 44, 12102(R) (1991).

${ }^{26}$ K. H. Satoh, S. Takeshita, A. Koda, R. Kadono, K. Ishida, S. Pyon, T. Sasagawa, and H. Takagi, Phys. Rev. B 77, 224503 (2008).

${ }^{27}$ Y. Ando, G. S. Boebinger, A. Passner, T. Kimura, and K. Kishio, Phys. Rev. Lett. 75, 4662 (1995).

${ }^{28}$ S. Ono, Y. Ando, T. Murayama, F. F. Balakirev, J. B. Betts, and G. S. Boebinger, Phys. Rev. Lett. 85, 638 (2000).

${ }^{29}$ H. Rifi, Z. Z. Li, S. Megtert, H. Raffy, O. Laborde, and P. Monceau, Physica C 235-240, 1433 (1994).

${ }^{30}$ Y. Ando, G. S. Boebinger, A. Passner, L. F. Schneemeyer, T. Kimura, M. Okuya, S. Watauchi, J. Shimoyama, K. Kishio, K. Tamasaku, N. Ichikawa, and S. Uchida, Phys. Rev. B 60, 12475 (1999).

${ }^{31}$ E. Helfand and N. R. Werthamer, Phys. Rev. 147, 288 (1966); N. R. Werthamer, E. Helfand, and P. C. Hohenberg, ibid. 147, 295 (1966).

${ }^{32}$ M. S. Osofsky, R. J. Soulen, S. A. Wolf, J. M. Broto, H. Rakoto, J. C. Ousset, G. Coffe, S. Askenazy, P. Pari, I. Bozovic, J. N. Eckstein, and G. F. Virshup, Phys. Rev. Lett. 71, 2315 (1993).

${ }^{33}$ S. I. Vedeneev, A. G. M. Jansen, E. Haanappel, and P. Wyder, Phys. Rev. B 60, 12467 (1999).

${ }^{34}$ A. P. Mackenzie, S. R. Julian, G. G. Lonzarich, A. Carrington, S. D. Hughes, R. S. Liu, and D. C. Sinclair, Phys. Rev. Lett. 71, 1238 (1993).

${ }^{35}$ A. Carrington, A. P. Mackenzie, and A. Tyler, Phys. Rev. B 54, R3788 (1996).

${ }^{36}$ In a recent paper, V. N. Zavaritsky, V. V. Kabanov, and A. S. Alexandrov, Europhys. Lett. 60, 127 (2002) address the controversy about the difference between resistively and thermodynamically determined $H_{c 2 \perp}(T)$. The authors who state that the resistive $H_{c 2 \perp}(T)$ is the critical field of the condensation of charged bosons, predict two anomalies in the specific heat, the lower- $T$ one corresponding to the resistive transition in a magnetic field.

${ }^{37}$ A. Schilling, R. Jin, J. D. Guo, and H. R. Ott, Phys. Rev. Lett. 71, 1899 (1993).

${ }^{38}$ Y. Yeshurun and A. P. Malozemoff, Phys. Rev. Lett. 60, 2202 (1988).

${ }^{39}$ S. I. Vedeneev, Cyril Proust, V. P. Mineev, M. Nardone, and G. L. J. A. Rikken, Phys. Rev. B 73, 014528 (2006).

${ }^{40}$ P. Li, F. F. Balakirev, and R. L. Greene, Phys. Rev. B 75, 172508 (2007).

${ }^{41}$ D. E. Prober, M. R. Beasley, and R. E. Schwall, Phys. Rev. B 15, 5245 (1977).

${ }^{42}$ Y. Hidaka and M. Suzuki, Nature (London) 338, 635 (1989).

${ }^{43}$ G. Blatter, V. B. Geshkenbein, and A. I. Larkin, Phys. Rev. Lett. 68, 875 (1992). 